\title{
Asymptotic correction of Numerov's eigenvalue estimates with general boundary conditions
}

\author{
Alan L. Andrew* \\ (Received 26 July 2001; revised 8 October 2002)
}

\begin{abstract}
The error in the estimate of the $k$ th eigenvalue of $-y^{\prime \prime}+$ $q y=\lambda y, y(0)=y(\pi)=0$, obtained by Numerov's method with uniform step length $h$, is $O\left(k^{6} h^{4}\right)$. The author and J. Paine showed that a correction technique of Paine, de Hoog and Anderssen reduced this to $O\left(k^{4} h^{5} / \sin (k h)\right)$, with negligible extra effort. Later the author extended the method to deal with boundary conditions of the form $y^{\prime}(a)=0$. This paper shows how a similar increase in accuracy can be obtained, with a little more effort, for problems with one or more boundary conditions of the form $y^{\prime}(a)=\alpha y(a)$ where $\alpha \neq 0$.
\end{abstract}

*Mathematics Department, La Trobe University, Victoria 3086, AUSTRALIA. mailto:a.andrew@latrobe.edu.au

${ }^{0}$ See http://anziamj.austms.org.au/V44/CTAC2001/Andr for this article, (C) Austral. Mathematical Soc. 2003. Published 1 April 2003, amended April 11, 2003. ISSN 1446-8735 


\section{Contents}

1 Introduction

C2

2 The finite difference equations

C4

3 Computing the correction

C9

4 Numerical results

C11

References

C16

\section{Introduction}

This paper proposes and tests an extension of a technique, first studied in $[21,23]$ for numerical solution of the regular Sturm-Liouville problem

$$
\begin{aligned}
& -y^{\prime \prime}+q y=\lambda y, \\
& y(0)=y(\pi)=0 .
\end{aligned}
$$

When the classical second order centred finite difference method, with $n$ equal subintervals, is used to solve (1-2), the error in the computed value of the $k$ th eigenvalue, $\lambda_{k}$, is $O\left(k^{4} h^{2}\right)$, where $h=$ $\pi / n$. This suggests that the method is unlikely to be useful for computing more than the first few eigenvalues. Nevertheless, Paine, de Hoog and Anderssen [21, 23] devised an implementation of the method which is remarkably efficient for computing a long string of eigenvalues, provided $q$ does not vary too rapidly. They used the fact that the first term in the asymptotic expansion (as $k \rightarrow$ $\infty)$ of the error in the computed value of $\lambda_{k}$ is independent of $q$. For this reason, the author [3] called their technique "asymptotic 
correction", but it has also been called "algebraic correction" [15] or "the AAdHP correction" [24, 18]. All that is required is the addition of a "correction", which depends only on $k$ and $h$ and not on $q$, to the finite difference estimate of $\lambda_{k}$ obtained for general $q$. The correction is calculated so that, in the special case of constant $q$, the estimate would equal the exact value of $\lambda_{k}$. Calculation of the correction is trivial for this problem, because, when $q$ is constant, the error is known in closed form. It is shown in [23] that, if $q \in C^{2}[0, \pi]$ and $k h$ is "sufficiently small", the error in the corrected estimate is only $O\left(k h^{2}\right)$.

Subsequently, asymptotic correction was shown to be useful for other methods $[12,13]$ and other boundary conditions $[1,4]$. Results have been proved which do not require the restriction to "sufficiently small" $k h$, and numerical results show that the method is generally useful for all eigenvalues (not just the higher ones) and that it can also be very effective for partial differential equations [3, 15]. Asymptotic correction has proved especially useful for the numerical solution of inverse eigenvalue problems [7, 15, 16, 20,22]. Results on asymptotic correction up to 1992 are reviewed in [6] and subsequent developments in [10].

A better method for (1-2) than the classical second order method is Numerov's method, which gives fourth order convergence while still using tridiagonal matrices. It was shown in [12] that asymptotic correction reduced the normal $O\left(k^{6} h^{4}\right)$ error for Numerov's method to $O\left(k^{4} h^{5} / \sin (k h)\right)$ without the restriction to "sufficiently small" $k h$. An extension of the method to problems with boundary conditions

$$
\begin{aligned}
& y^{\prime}(0)=y^{\prime}(\pi)=0, \\
& y(0)=y^{\prime}(\pi)=0, \\
& \text { or } \quad y^{\prime}(0)=y(\pi)=0,
\end{aligned}
$$


was described in [9]. It was noted there that there were two difficulties to be overcome before the method could be used for problems with one or both boundary conditions of the form $y^{\prime}(a)=\alpha y(a)$ with $\alpha \neq 0$ : (i) the eigenvalue parameter enters the finite difference equations quadratically, and (ii) the correction can no longer be computed in closed form. By dealing with these difficulties in Sections 2 and 3 respectively, this paper completes the extension of the corrected Numerov method to general separated boundary conditions. Section 4 investigates its numerical performance.

\section{The finite difference equations}

Numerov's method approximates (1) by the three term recurrence relation

$$
\begin{aligned}
& -\left[12-h^{2} q\left(x_{i-1}\right)\right] y_{i-1}+\left[24+10 h^{2} q\left(x_{i}\right)\right] y_{i} \\
& \quad-\left[12-h^{2} q\left(x_{i+1}\right)\right] y_{i+1}=h^{2} \Lambda\left[y_{i-1}+10 y_{i}+y_{i+1}\right] .
\end{aligned}
$$

Here $\Lambda$ and $y_{i}$ are the approximations of $\lambda$ and $y\left(x_{i}\right)$, where $x_{i}=i h$. To achieve fourth order convergence for problems with first order derivatives in the boundary conditions, it is necessary to have a suitable fourth order approximation to the first derivative.

In [19], the boundary condition

$$
y^{\prime}(0)=\alpha_{1} y(0)
$$

was approximated by the fourth order scheme

$$
\left(6-h^{2} q(-h)\right) y_{-1}+12 h \alpha_{1} y_{0}-\left(6-h^{2} q(h)\right) y_{1}=\Lambda h^{2}\left(-y_{-1}+y_{1}\right) \text {. }
$$

A disadvantage of (8) is that, even when $\alpha_{1}=0$, combination with (6) leads to a matrix eigenvalue problem nonlinear in the eigenvalue. This was not a serious problem in [19] where the difference 
equations were solved by shooting, which is quite appropriate as, in the absence of asymptotic correction, the method is mainly useful for computing just a small number of eigenvalues. However, the main advantage of asymptotic correction is that it enables a large number of eigenvalues to be computed efficiently. When this is our aim, shooting is less satisfactory as it requires a separate calculation for each eigenvalue, whereas there are many good methods for simultaneous computation of all eigenvalues of the matrix eigenvalue problem.

For this reason, (7) was approximated in [9] by the alternative fourth order difference scheme

$$
y_{-1}+h\left[\alpha_{1}\left(2+(q(0)-\Lambda) h^{2} / 3\right)+h^{2} q^{\prime}(0) / 3\right] y_{0}-y_{1}=0,
$$

whereas the boundary condition

$$
y^{\prime}(\pi)=\alpha_{2} y(\pi)
$$

was approximated by

$$
y_{n-1}+h\left[\alpha_{2}\left(2+(q(\pi)-\Lambda) h^{2} / 3\right)+h^{2} q^{\prime}(\pi) / 3\right] y_{n}-y_{n+1}=0 .
$$

Combining (9) with (6) with $i=0$ gives the equation

$$
\begin{aligned}
& \left\{h\left[12-h^{2} q(-h)\right]\left[\alpha_{1}\left(2+q(0) h^{2} / 3\right)+q^{\prime}(0) h^{2} / 3\right]\right. \\
& \left.\quad+24+10 h^{2} q(0)\right\} y_{0}-\left[24-h^{2}(q(-h)+q(h))\right] y_{1} \\
& +h^{2} \Lambda\left\{\left[h \alpha_{1}\left(-2+h^{2}(q(-h)+q(0)) / 3\right)+h^{3} q^{\prime}(0) / 3-10\right] y_{0}\right. \\
& \left.\quad-2 y_{1}\right\}-\left(h^{2} \Lambda\right)^{2}\left(h \alpha_{1} / 3\right) y_{0}=0,
\end{aligned}
$$

while combining (11) with (6) with $i=n$ gives

$$
\begin{aligned}
& -\left[24-h^{2}(q(\pi-h)+q(\pi+h))\right] y_{n-1}+\left\{24+10 h^{2} q(\pi)\right. \\
& \left.-h\left[12-h^{2} q(\pi+h)\right]\left[2 \alpha_{2}+\alpha_{2} q(\pi) h^{2} / 3+h^{2} q^{\prime}(\pi) / 3\right]\right\} y_{n} \\
& +h^{2} \Lambda\left\{-2 y_{n-1}+\left[-10+h \alpha_{2}\left(2-h^{2}(q(\pi)+q(\pi+h)) / 3\right)\right.\right. \\
& \left.\left.\quad-h^{3} q^{\prime}(\pi) / 3\right] y_{n}\right\}+\left(h^{2} \Lambda\right)^{2}\left(h \alpha_{2} / 3\right) y_{n}=0 .
\end{aligned}
$$


In the case $\alpha_{1}=\alpha_{2}=0$, considered in [9], (12) and (13) simplify and, together with (6) for $i=1, \ldots, n-1$, give the matrix equation

$$
A_{0} \mathbf{y}+h^{2} \Lambda A_{1} \mathbf{y}=0
$$

where $\mathbf{y}=\left(y_{0}, y_{1}, \ldots, y_{n}\right)^{T}$ and the $(n+1) \times(n+1)$ matrices $A_{0}$ and $A_{1}$ are tridiagonal. Hence computing the Numerov eigenvalue estimates requires essentially the same computational effort for the boundary conditions (3), (4) and (5) as for the boundary conditions (2). Moreover, with these boundary conditions, asymptotic correction again requires negligible computational effort, as the error with constant $q$ is known in closed form. For details, see [9], which also reports the results of some numerical tests. Additional numerical results are included in [10].

The computation is less simple when $\alpha_{1}$ and $\alpha_{2}$ are not both zero. Not only is the error for constant $q$ no longer known in closed form, but, even when (9) and (11) are used, the matrix eigenvalue problem to be solved is not (14), but a more difficult problem of the form

$$
A_{0} \mathbf{y}+h^{2} \Lambda A_{1} \mathbf{y}+\left(h^{2} \Lambda\right)^{2} A_{2} \mathbf{y}=0 .
$$

Nevertheless, we still found the method described here to be significantly more efficient than the one described in [1]. Although "quadratic eigenvalue problems" like (15) require more computational effort than the classical problem (14), there are many good methods available for their numerical solution, and indeed for the numerical solution of eigenvalue problems with rather general nonlinear dependence on the eigenvalue parameter [8]. This is important for the viability of several standard methods for the numerical solution of (1), and other problems linear in the eigenvalue, which require the solution of a matrix eigenvalue problem with nonlinear dependence on the eigenvalue parameter [8]. A good survey (with over 150 references) dealing specifically with the quadratic eigenvalue problem is given in [25]. 
The most commonly used method for the numerical solution of equations of the form of (15) is to set $\mathbf{z}=h^{2} \Lambda \mathbf{y}$ and write (15) as

$$
\left(\begin{array}{cc}
A_{1} & A_{0} \\
I & 0
\end{array}\right)\left(\begin{array}{l}
\mathbf{z} \\
\mathbf{y}
\end{array}\right)=h^{2} \Lambda\left(\begin{array}{cc}
-A_{2} & 0 \\
0 & I
\end{array}\right)\left(\begin{array}{l}
\mathbf{z} \\
\mathbf{y}
\end{array}\right)
$$

which may be solved by the same methods as (14). Usually the main disadvantage of this method is that it doubles the dimensions of the matrices. The factor by which this multiplies the computational effort required is typically about 8 for dense matrices. For sparse matrices, the factor depends on the sparsity pattern and the method used, but is usually at least 4 . A potentially more serious disadvantage of using (16) for our problem is that $A_{2}$ is singular. All its elements are zero except perhaps the first diagonal element (which is nonzero if $\alpha_{1} \neq 0$ ) and the last diagonal element (which is nonzero if $\alpha_{2} \neq 0$ ). Solving the problem as (16) would produce a (spurious) infinite eigenvalue of multiplicity at least $n-1$.

A simple modification of the classical "linearisation" procedure described in the last paragraph is better for our problem. Instead of doubling the number of components of the vector $\mathbf{y}$, we simply add one component for each row in which the eigenvalue occurs quadratically. When $\alpha_{1} \alpha_{2} \neq 0$, for example, the matrices $A_{i}, i=$ $0,1,2$, should be partitioned as $\left(\mathbf{a}_{i 0}, \hat{A}_{i}, \mathbf{a}_{i n}\right)$, where $\mathbf{a}_{i 0}$ and $\mathbf{a}_{i n}$ are the first and last columns respectively of $A_{i}$, and $\hat{A}_{i}$ is the matrix obtained from $A_{i}$ by deleting these columns. Then $\hat{A}_{2}=0, \mathbf{a}_{20}=$ $-\left(h \alpha_{1} / 3\right) \mathbf{e}_{1}$ and $\mathbf{a}_{2 n}=\left(h \alpha_{2} / 3\right) \mathbf{e}_{n+1}$, where $\mathbf{e}_{i}$ is the $i$ th column of the $(n+1) \times(n+1)$ identity matrix, and (15) can be written as

$$
A \mathbf{x}+h^{2} \Lambda B \mathbf{x}=0,
$$

where

$$
A=\left(\begin{array}{ccccc}
\mathbf{a}_{00} & \hat{A}_{0} & \mathbf{a}_{0 n} & \mathbf{0} & \mathbf{0} \\
0 & \mathbf{0}^{T} & 0 & 1 & 0 \\
0 & \mathbf{0}^{T} & 0 & 0 & 1
\end{array}\right)
$$




$$
B=\left(\begin{array}{ccccc}
\mathbf{a}_{10} & \hat{A}_{1} & \mathbf{a}_{1 n} & \mathbf{a}_{20} / h & \mathbf{a}_{2 n} / h \\
-h & \mathbf{0}^{T} & 0 & 0 & 0 \\
0 & \mathbf{0}^{T} & -h & 0 & 0
\end{array}\right)
$$

and $\mathbf{x}^{T}=\left(\mathbf{y}^{T}, \Lambda h^{3} y_{0}, \Lambda h^{3} y_{n}\right)$. One disadvantage that this linearisation shares with the classical scheme (16) is that the tridiagonal structure of one of the matrices is lost. However, it is still sparse and more "nearly" tridiagonal than the first matrix in (16). This sparsity could be used to compute the eigenvalues of (17) more efficiently, but this was not done in the calculations reported here which, like those in $[9,10]$, simply used the MATLAB command EIG(A,B), because the corrected Numerov method produced very good results even with quite a coarse mesh. (Much finer meshes are used for the same problem in [1] and [17].) Moreover, the $(m+3) \times(m+3)$ matrices $A$ and $B$ are significantly smaller than those produced by the linearisation (16).

The above procedure is readily adapted to deal with other separated boundary conditions. When $\alpha_{1} \alpha_{2}=0$, using the same computer code as for $\alpha_{1} \alpha_{2} \neq 0$ would lead to a singular $B$ and, although this may not be fatal (see the discussion of small $\alpha_{i}$ in Section 4), it is better to proceed as follows. If the boundary conditions are (7), (10) with $\alpha_{1}=0 \neq \alpha_{2}$ for example, the second last row and column of $A$ and $B$ (and the second last element of $\mathbf{x}$ ) should be deleted. Similarly, if $\alpha_{1} \neq 0=\alpha_{2}$ then the last row and column of $A$ and $B$ (and the last element of $\mathbf{x}$ ) should be deleted. For boundary conditions $y(0)=0, y^{\prime}(\pi)=\alpha_{2} y(\pi), \alpha_{2} \neq 0$, the first and the second last rows and columns of $A$ and $B$ should be deleted, while for boundary conditions $y^{\prime}(0)=\alpha_{1} y(0), y(\pi)=0, \alpha_{1} \neq 0$, the third last and the last rows and columns of $A$ and $B$ should be deleted. The remaining separated boundary conditions, (2), (3), (4) and (5) are dealt with in [12] and [9]. (Of course, all results given here for the interval $[0, \pi]$ are readily generalised to an arbitrary finite interval, as in [4].) 


\section{Computing the correction}

The method described in Section 2 will give results with $O\left(k^{6} h^{4}\right)$ error in the computed estimate, $\Lambda_{k}^{(n)}$, of the $k$ th eigenvalue, $\lambda_{k}$. Asymptotic correction reduces the growth in the error with increasing $k$ by replacing $\Lambda_{k}^{(n)}$, by the "corrected" estimate $\tilde{\Lambda}_{k}^{(n)}=$ $\Lambda_{k}^{(n)}+\varepsilon_{k}^{(n)}$, where $\varepsilon_{k}^{(n)}$ is computed so that the error is zero when $q$ is constant. Although closed form solutions like those of $[9,12]$ are not available for the problems considered here, $\varepsilon_{k}^{(n)}$ is easily computed by a variant of a method proposed in $[1,2]$ and considered further in [14]. For most $k$ (and all $k$ if $\alpha_{1}>0>\alpha_{2}$ ), it was shown there that, when $q=0, \lambda_{k}$ is the square of the limit of the sequence $\left\{\mu_{k}^{(j)}\right\}_{j=0}^{\infty}$ defined by

$$
\mu_{k}^{(j)}=k-1+f_{1}\left(\mu_{k}^{(j-1)}\right), \quad \mu_{k}^{(0)}=k-1,
$$

where

$$
f_{1}(z)=\left[\sin ^{-1}\left(\alpha_{1} /\left(\alpha_{1}^{2}+z^{2}\right)^{1 / 2}\right)-\sin ^{-1}\left(\alpha_{2} /\left(\alpha_{2}^{2}+z^{2}\right)^{1 / 2}\right)\right] / \pi .
$$

The case $\alpha_{1} \alpha_{2} \neq 0$ was emphasized in [1], but, when $\alpha_{1} \alpha_{2}=0$, the initial condition in (18) produces the indeterminate $0 / 0$ in (19) when $k=0$. This can be avoided by setting $\mu_{k}^{(0)}=k-1-h$. An alternative slightly simpler scheme, not mentioned in [1,2], which appears to converge at the same rate (modulo roundoff) to the same limit, when $\alpha_{1} \alpha_{2} \neq 0$, is

$$
\mu_{k}^{(j)}=K+\left[\tan ^{-1}\left(\mu_{k}^{(j-1)} / \alpha_{2}\right)-\tan ^{-1}\left(\mu_{k}^{(j-1)} / \alpha_{1}\right)\right] / \pi,
$$

where

$$
K=k-1+\left(\operatorname{sign}\left(\alpha_{1}\right)-\operatorname{sign}\left(\alpha_{2}\right)\right) / 2,
$$

so that $K=k$ when $\alpha_{1}>0>\alpha_{2}$. It was shown in [9] that, for all real numbers $\theta$ and $\beta, y_{i}=\sin (i \theta+\beta)$ satisfies (6) for all integers $i$, 
when $q=0$ and

$$
\Lambda=\frac{12\left[\sin ^{2}(\theta / 2)\right]}{h^{2}\left[3-\sin ^{2}(\theta / 2)\right]} .
$$

Substitution of this form for $y_{i}$ in (9) and (11), as in [9], gives an equation to be solved for $\theta$ and hence $\Lambda_{k}^{(n)}$ (for $q=0$ ) and $\varepsilon_{k}^{(n)}$. The equation for $\theta$ may be solved by an iteration similar to (18) or (20). When $\alpha_{1} \alpha_{2} \neq 0$, this gives approximations $\tilde{\Lambda}_{k}^{(n)}(N)$ to $\tilde{\Lambda}_{k}^{(n)}$ defined, after simplification, by

$$
\tilde{\Lambda}_{k}^{(n)}(N)=\Lambda_{k}^{(n)}+\left(\mu_{k}^{(N)}\right)^{2}-12 \sin ^{2}\left(\theta_{k}^{(N)} / 2\right) /\left(h^{2}\left(3-\sin ^{2}\left(\theta_{k}^{(N)} / 2\right)\right)\right)
$$

where

$$
\theta_{k}^{(0)}=K h-h^{2}
$$

$K$ is defined in (21), and, for $j=1, \ldots, N$,

$$
\begin{aligned}
\theta_{k}^{(j)}= & {\left[\tan ^{-1}\left(\sin \left(\theta_{k}^{(j-1)}\right) /\left(h \alpha_{2} f\left(\theta_{k}^{(j-1)}\right)\right)\right)\right.} \\
& \left.-\tan ^{-1}\left(\sin \left(\theta_{k}^{(j-1)}\right) /\left(h \alpha_{1} f\left(\theta_{k}^{(j-1)}\right)\right)\right)\right] / n+h K,
\end{aligned}
$$

where

$$
f(\theta)=(3+3 \cos (\theta)) /(5+\cos (\theta)) .
$$

In practice, it did not seem to matter whether (18) or (20) was used to compute $\mu_{k}^{(N)}$ in (23), since the results obtained by the two alternative definitions usually agreed to at least 10 significant figures. The relationship between $\tilde{\Lambda}_{k}^{(n)}(N)$ and $\tilde{\Lambda}_{k}^{(n)}$ is discussed in the next section. A similar method may be used in the remaining four cases, where $\alpha_{1} \alpha_{2} \neq 0$ :

1. $y^{\prime}(0)-\alpha_{1} y(0)=y(\pi)=0$;

2. $y^{\prime}(0)-\alpha_{1} y(0)=y^{\prime}(\pi)=0$;

3. $y(0)=y^{\prime}(\pi)-\alpha_{2} y(\pi)=0$; and 
4. $y^{\prime}(0)=y^{\prime}(\pi)-\alpha_{2} y(\pi)=0$.

Each case requires a separately coded formula for computing $\theta_{k}^{(j)}$. See the discussion of this and some related matters in [11].

\section{Numerical results}

The numerical results in [1, 2] for (1) all used the example $q(x)=e^{x}$ with boundary conditions $y^{\prime}(0)-y(0)=y^{\prime}(\pi)+y(\pi)=0$, which is equivalent to setting $\alpha_{1}=1$ and $\alpha_{2}=-1$ in (7) and (10). To assist comparison of our method with the method of [1], we used the same example in our numerical calculations. We calculated $\Lambda_{k}^{(n)}$ and $\tilde{\Lambda}_{k}^{(n)}$ for $n=10,20,40,80,120,150,160$ and 200 , and $k=1, \ldots, n+1$. Some representative results are shown in Table 1. To test (21), some calculations were made with $\alpha_{i}$ of different sign.

It was conjectured in [9], on the basis of numerical results, and results proved in [12] for problems with boundary conditions (2), that

$$
\lambda_{k}-\tilde{\Lambda}_{k}^{(n)}=O\left(k^{4} h^{5} / \sin \left(\left(k-\frac{1}{2}\right) h\right)\right) .
$$

Our results for the above example, like the results of [10], provide further support for this conjecture. The regularity of the scaled errors (which are multiplied by 100 in Table 1 for ease of tabulation) is demonstrated by the last four columns of Table 1. This regularity allows higher accuracy to be obtained by extrapolation of the corrected eigenvalues. Indeed the "exact" $\lambda_{k}$ used for calculating the errors in Table 1 were computed as $C_{k}(200,150)$, using 
TABle 1: Results for (1), (7), (10) with $q(x)=e^{x}, \alpha_{1}=1, \alpha_{2}=-1$

\begin{tabular}{|c|c|c|c|c|c|c|c|}
\hline \multirow[b]{2}{*}{$k$} & \multirow[b]{2}{*}{$\lambda_{k}$} & \multirow[b]{2}{*}{$\lambda_{k}-\Lambda_{k}^{(40)}$} & \multirow[b]{2}{*}{$\lambda_{k}-\tilde{\Lambda}_{k}^{(40)}$} & \multicolumn{4}{|c|}{$100\left(\lambda_{k}-\tilde{\Lambda}_{k}^{(n)}\right) \sin \left(\left(k-\frac{1}{2}\right) h\right) /\left(k^{4} h^{5}\right)$} \\
\hline & & & & $n=10$ & $n=20$ & $n=40$ & $n=80$ \\
\hline 1 & 3.33457 & $3.16 \mathrm{E}-7$ & $2.92 \mathrm{E}-7$ & 0.40 & 0.39 & 0.38 & 0.38 \\
\hline 2 & 7.95107 & $2.07 \mathrm{E}-5$ & $1.87 \mathrm{E}-5$ & 4.62 & 4.60 & 4.60 & 4.60 \\
\hline 3 & 13.2643 & $1.05 \mathrm{E}-4$ & $7.54 \mathrm{E}-5$ & 5.93 & 6.04 & 6.08 & 6.09 \\
\hline 4 & 19.0014 & $3.39 \mathrm{E}-4$ & $1.34 \mathrm{E}-4$ & 4.44 & 4.68 & 4.75 & 4.77 \\
\hline 5 & 25.5680 & $1.23 \mathrm{E}-3$ & $3.03 \mathrm{E}-4$ & 4.47 & 5.39 & 5.62 & 5.68 \\
\hline 6 & 34.0653 & $3.82 \mathrm{E}-3$ & $6.75 \mathrm{E}-4$ & 4.75 & 6.76 & 7.30 & 7.43 \\
\hline 7 & 44.7892 & $9.95 \mathrm{E}-3$ & $1.14 \mathrm{E}-3$ & 4.04 & 6.92 & 7.76 & 7.98 \\
\hline 8 & 57.6418 & $2.30 \mathrm{E}-2$ & $1.67 \mathrm{E}-3$ & 3.09 & 6.46 & 7.58 & 7.87 \\
\hline 9 & 72.5554 & $4.87 \mathrm{E}-2$ & $2.27 \mathrm{E}-3$ & 2.47 & 5.80 & 7.17 & 7.53 \\
\hline 10 & 89.5004 & $9.57 \mathrm{E}-2$ & $2.95 \mathrm{E}-3$ & 1.39 & 5.10 & 6.70 & 7.13 \\
\hline 11 & 108.463 & $1.76 \mathrm{E}-1$ & $3.71 \mathrm{E}-3$ & 2.16 & 4.42 & 6.22 & 6.72 \\
\hline 12 & 129.436 & 3.09E-1 & $4.54 \mathrm{E}-3$ & & 3.78 & 5.76 & 6.32 \\
\hline 13 & 152.417 & $5.16 \mathrm{E}-1$ & $5.46 \mathrm{E}-3$ & & 3.20 & 5.32 & 5.95 \\
\hline 14 & 177.402 & 8.31E-1 & $6.47 \mathrm{E}-3$ & & 2.70 & 4.92 & 5.60 \\
\hline 15 & 204.390 & $1.29 \mathrm{E} 0$ & $7.56 \mathrm{E}-3$ & & 2.27 & 4.54 & 5.29 \\
\hline 16 & 233.381 & $1.96 \mathrm{E} 0$ & $8.74 \mathrm{E}-3$ & & 1.92 & 4.19 & 4.99 \\
\hline 17 & 264.373 & $2.89 \mathrm{E} 0$ & $1.00 \mathrm{E}-2$ & & 1.68 & 3.86 & 4.72 \\
\hline 18 & 297.367 & $4.16 \mathrm{E} 0$ & $1.14 \mathrm{E}-2$ & & 1.57 & 3.56 & 4.48 \\
\hline 19 & 332.362 & $5.87 \mathrm{E} 0$ & $1.28 \mathrm{E}-2$ & & 1.69 & 3.28 & 4.24 \\
\hline 20 & 369.358 & $8.15 \mathrm{E} 0$ & $1.44 \mathrm{E}-2$ & & 1.36 & 3.01 & 4.03 \\
\hline 21 & 408.354 & $1.11 \mathrm{E} 1$ & $1.61 \mathrm{E}-2$ & & 2.85 & 2.77 & 3.83 \\
\hline
\end{tabular}


the extrapolation formula

$$
C_{k}(n, m)=\frac{n^{5} \sin \left(\left(k-\frac{1}{2}\right) \pi / n\right) \tilde{\Lambda}_{k}^{(n)}-m^{5} \sin \left(\left(k-\frac{1}{2}\right) \pi / m\right) \tilde{\Lambda}_{k}^{(m)}}{n^{5} \sin \left(\left(k-\frac{1}{2}\right) \pi / n\right)-m^{5} \sin \left(\left(k-\frac{1}{2}\right) \pi / m\right)}
$$

suggested by $(27)$. Comparison with $C_{k}(160,120)$ suggests that all figures shown in Table 1 are correct. See also the discussion in $[6,9,10]$ on combining asymptotic correction with extrapolation. The method described here is to be preferred to the method of [1] because of its more rapid convergence. Even for $n=40$ it already gives very good results for the lower eigenvalues. However, for the achievement of a specified accuracy, the corrected Numerov method still requires more computational effort for the boundary conditions considered here than for (2), (3), (4) and (5). The first reason is that the matrices in (17) are not only marginally larger than those required for the other boundary conditions for the same mesh size, but are also not both tridiagonal. Note also that, although the matrices are $(n+3) \times(n+3)$, only $n+1$ useful eigenvalue estimates are obtained. The other two eigenvalues of (17) are spurious. The production of spurious eigenvalues is a feature of many numerical methods (see [5] for some references). With some numerical methods, spurious eigenvalues can be difficult to separate from the genuine ones. No such difficulty arises in this case, as the spurious eigenvalues, which are related to the very small entries in the last two rows of $B$, have much greater magnitude than the true ones. As one test of whether the large eigenvalues led to instability, we also calculated the eigenvalues as the reciprocals of the eigenvalues, $\sigma$, of the problem $B x=\sigma A x$. The results were almost identical. Since very small values of $\alpha_{1}$ or $\alpha_{2}$ will lead to even larger spurious eigenvalues, we also tested our method on the same problem but with $\alpha_{1}=-\alpha_{2}=10^{-10}$. The results converged as $n \rightarrow \infty$ to values close to those obtained by the method of [9] for the boundary conditions (3). With $\alpha_{1}=-\alpha_{2}=10^{10}$, the results converged to values 
very close to those obtained by the method of [12] for boundary conditions (2). These results all suggest that stability is not a serious problem, probably because of the robustness of the $Q Z$ algorithm for pencils with very large or even infinite eigenvalues [26].

The second reason why the method is less efficient for the boundary conditions considered here, is that computation of the appropriate correction $\varepsilon_{k}^{(n)}$ is no longer trivial, but requires an approximation scheme like (23). For all $k$ and $n$ we found asymptotic correction to improve the accuracy of the computed eigenvalues. However, the recommendation to use asymptotic correction must be more qualified than with boundary conditions (2), (3), (4) and (5), because of the computational cost of computing $\varepsilon_{k}^{(n)}$ by (23). As shown in Table 2, convergence of the iteration (23) is slowest for the lowest eigenvalues, which are precisely those for which the correction makes the smallest improvement. Also (18), (20) and (23) are unsatisfactory when $K<1$. When only the first one or two eigenvalues are required, asymptotic correction is recommended only when the boundary conditions are (2), (3), (4) or (5). For other boundary conditions, mesh refinement produces a greater improvement in these low eigenvalues for a given effort. When a large number of eigenvalues are required, asymptotic correction is recommended regardless of the boundary conditions. Moreover, since the correction is independent of $q$, a single computation of the correction is sufficient if solutions of (1), (7), (10) are required for several different $q$, with the same $\alpha_{1}$ and $\alpha_{2}$. Also, just as asymptotic correction has proved vital when used in conjunction with second order finite difference methods for the solution of inverse eigenvalue problems [20, 22], the same should remain true if Numerov's method is used, whatever the boundary conditions.

We computed $\tilde{\Lambda}_{k}^{(n)}$ not only as $\lim _{j \rightarrow \infty} \tilde{\Lambda}_{k}^{(n)}(j)$, using (23), but also by the slower method of using MATLAB to solve (17) directly in 
TABLE 2: Computing the correction for the same example

\begin{tabular}{|c|c|c|c|c|c|c|}
\hline \multirow[b]{2}{*}{$k$} & \multicolumn{4}{|c|}{$\lambda_{k}-\tilde{\Lambda}_{k}^{(20)}(N)$} & \multirow[t]{2}{*}{$\lambda_{k}-\tilde{\Lambda}_{k}^{(20)}$} & \multirow[t]{2}{*}{$\lambda_{k}-\Lambda_{k}^{(20)}$} \\
\hline & $N=2$ & $N=5$ & $N=10$ & $N=20$ & & \\
\hline 1 & $2.10 \mathrm{E}-1$ & $-2.18 \mathrm{E}-2$ & $4.14 \mathrm{E}-4$ & $4.88 \mathrm{E}-6$ & $4.73 \mathrm{E}-6$ & $5.12 \mathrm{E}-6$ \\
\hline 2 & $1.10 \mathrm{E}-1$ & $-8.02 \mathrm{E}-4$ & $3.02 \mathrm{E}-4$ & $3.02 \mathrm{E}-4$ & $3.02 \mathrm{E}-4$ & $3.34 \mathrm{E}-4$ \\
\hline 3 & $3.91 \mathrm{E}-2$ & $1.18 \mathrm{E}-3$ & $1.22 \mathrm{E}-3$ & $1.22 \mathrm{E}-3$ & $1.22 \mathrm{E}-3$ & $1.70 \mathrm{E}-3$ \\
\hline 4 & $1.79 \mathrm{E}-2$ & $2.19 \mathrm{E}-3$ & $2.19 \mathrm{E}-3$ & $2.19 \mathrm{E}-3$ & $2.19 \mathrm{E}-3$ & $5.50 \mathrm{E}-3$ \\
\hline 10 & $4.99 \mathrm{E}-2$ & $4.89 \mathrm{E}-2$ & $4.89 \mathrm{E}-2$ & $4.89 \mathrm{E}-2$ & $4.89 \mathrm{E}-2$ & $1.60 \mathrm{E} 0$ \\
\hline 21 & $-1.55 \mathrm{E} 1$ & $-1.55 \mathrm{E} 1$ & $-1.55 \mathrm{E} 1$ & $-1.55 \mathrm{E} 1$ & $-8.72 \mathrm{E} 0$ & $1.51 \mathrm{E} 2$ \\
\hline
\end{tabular}

the case $q=0$, still computing $\lim _{j \rightarrow \infty} \mu_{k}^{(j)}$ by (18) or (20). For all $k \leq n$, the two results agreed to at least 10 significant figures, and for $k>5$ no more than 5 iterations were required for an excellent estimate. However, we found that

$$
\lim _{j \rightarrow \infty} \theta_{n+1}^{(j)}=\lim _{j \rightarrow \infty} \theta_{n-1}^{(j)} \quad \text { and hence } \quad \lim _{j \rightarrow \infty} \tilde{\Lambda}_{n+1}^{(n)}(j) \neq \tilde{\Lambda}_{n+1}^{(n)} .
$$

A similar problem affects the iterative scheme suggested for the second order method in [1], though this was not mentioned in [1], presumably because it is recommended that calculations should use $n \geq 2 k$ if $\lambda_{k}$ is required. The values of $\tilde{\Lambda}_{n+1}^{(n)}$ shown in our tables are the exact values, not the values given by (23). The reason for (29) is that (23) is based on the assumption that the eigenvectors of (15) have the form $y_{i}=\sin (i \theta+\beta)$ for some constants $\beta$ and $\theta$. This assumption is true for most, but not all, of the eigenvectors. When $\alpha_{1}>0>\alpha_{2}$ it is true for those which correspond to $\lambda_{1}, \ldots, \lambda_{n}$, but it is not true for those corresponding to $\lambda_{n+1}$ and the two spurious eigenvalues. Nevertheless, $\lim _{j \rightarrow \infty} \tilde{\Lambda}_{n+1}^{(n)}(j)$ was still a sufficiently good approximation to $\tilde{\Lambda}_{n+1}^{(n)}$ to produce a significant improvement in the uncorrected Numerov estimate, and our results show that, if $\lim _{j \rightarrow \infty} \tilde{\Lambda}_{n+1}^{(n)}(j)$ were used instead of $\tilde{\Lambda}_{n+1}^{(n)},(27)$ would still be satisfied. 


\section{References}

[1] R. S. Anderssen and F. R. de Hoog. On the correction of finite difference eigenvalue approximations for Sturm-Liouville problems with general boundary conditions, BIT, 24: 401-412, 1984. C3, C6, C8, C9, C11, C13, C15

[2] R. S. Anderssen and F. R. de Hoog. On the correction of finite difference eigenvalue approximations for Sturm-Liouville problems with general boundary conditions. Australian National University Report CMA-R05-82, 1982. C9, C11

[3] A. L. Andrew. Asymptotic correction of finite difference eigenvalues. In J. Noye and R. May, editors, Computational techniques and applications: CTAC-85, pages 333-341, Amsterdam, 1986 North-Holland. C2, C3

[4] A. L. Andrew. Correction of finite element eigenvalues for problems with natural or periodic boundary conditions. BIT, 28:254-269, 1988. C3, C8

[5] A. L. Andrew. Some recent developments in finite element eigenvalue computation. In J. Noye and C. Fletcher, editors, Computational techniques and applications: CTAC-87, pages 83-91, Amsterdam, 1988. North-Holland. C13

[6] A. L. Andrew. Asymptotic correction of computed eigenvalues of differential equations. Ann. Numer. Math., 1: 41-51, 1994. C3, C13

[7] A. L. Andrew. Some recent developments in inverse eigenvalue problems. In D. Stewart, D. Singleton and H. Gardner, editors, Computational techniques and applications: CTAC-93, pages 94-102, Singapore, 1994. World Scientific. C3 
[8] A. L. Andrew. Solution of nonlinear and multiparameter eigenvalue problems. In R. L. May and A. K. Easton, editors, Computational techniques and applications: CTAC-95, pages 97-104, Singapore, 1996. World Scientific. C6

[9] A. L. Andrew. Asymptotic correction of Numerov's eigenvalue estimates with natural boundary conditions. J. Comput. Appl. Math., 125: 359-366, 2000. C4, C5, C6, C8, C9, C10, C11, $\mathrm{C} 13$

[10] A. L. Andrew. Twenty years of asymptotic correction for eigenvalue computation. ANZIAM J., 42(E): C96-C116, 2000. [Online] http://anziamj . austms . org. au/V42/CTAC99/Andr. C3, C6, C8, C11, C13

[11] A. L. Andrew. Asymptotic correction of more Sturm-Liouville eigenvalue estimates. BIT, to appear. C11

[12] A. L. Andrew and J. W. Paine. Correction of Numerov's eigenvalue estimates. Numer. Math., 47: 289-300, 1985. C3, C8, C9, C11, C14

[13] A. L. Andrew and J. W. Paine. Correction of finite element estimates for Sturm-Liouville eigenvalues. Numer. Math., 50: 205-215, 1986. C3

[14] F. R. de Hoog and R. S. Anderssen. Asymptotic formulas for discrete eigenvalue problems in Liouville normal form. Math. Mod. Meth. Appl. Sci., 11: 43-56, 2001. C9

[15] C. R. Dun. Algebraic correction methods for two-dimensional eigenvalue problems. $\mathrm{PhD}$ thesis, Australian National University, Canberra, Australia, 1995. C3 
[16] R. H. Fabiano, R. Knobel and B. D. Lowe. A finite difference algorithm for an inverse Sturm-Liouville problem. IMA J. Numer. Anal., 15: 75-88, 1995. C3

[17] P. Ghelardoni, G. Gheri and M. Marletta. Spectral corrections for Sturm-Liouville problems. J. Comput. Appl. Math., 132: 443-459, 2001. C8

[18] P. Kosowski. The relative error in the Pruess method for Sturm-Liouville problems. Linear Algebra Appl., 309: 325-337, 2000. C3

[19] J. P. Leroy and R. Wallace. Renormalized Numerov method applied to eigenvalue problems: extensions to include single derivative terms and a variety of boundary conditions. J. Phys. Chem., 89: 1928-1932, 1985. C4

[20] J. T. Marti. Small potential corrections for the discrete eigenvalues of the Sturm-Liouville problem. Numer. Math., 57: 51-62, 1990. C3, C14

[21] J. W. Paine. Numerical approximation of Sturm-Liouville eigenvalues. PhD thesis, Australian National University, Canberra, Australia, 1979. C2

[22] J. Paine. A numerical method for the inverse Sturm-Liouville problem. SIAM J. Sci. Stat. Comput., 5: 149-156, 1984. C3, C14

[23] J. W. Paine, F. R. de Hoog and R. S. Anderssen. On the correction of finite difference eigenvalue approximations for Sturm-Liouville problems. Computing, 26: 123-139, 1981. $\mathrm{C} 2, \mathrm{C} 3$

[24] J. D. Pryce. Numerical solution of Sturm-Liouville problems. Oxford Univ. Press, London, 1993. C3 
[25] F. Tisseur and K. Meerbergen. The quadratic eigenvalue problem. SIAM Rev., 43: 235-286, 2001. C6

[26] D. S. Watkins. Performance of the $Q Z$-algorithm in the presence of infinite eigenvalues. SIAM J. Matrix Anal. Appl., 22: $364-375,2000$. C14 